\title{
Research on Smart Substation-Area Protection Based on Multi-Agent Technology
}

\author{
LIU Penghui ${ }^{1, a}$,LIU Yuanlong ${ }^{2}$ \\ ${ }^{1}$ College of Automation Engineering, Qingdao University, Qingdao 266071, China \\ ${ }^{2}$ Shandong Electric Power Dispatching Control Center, Jinan 250001,China \\ aemail:981467500@qq.com
}

Key words: agent; substation-area ; MAS; smart; protection

\begin{abstract}
In view of the present smart substation-area protection situation which exists some traditional relay protection problems, this paper put forward a kind of smart substation-area protection based on multi-Agent technology, which combines with the characteristics of the Agent in the relay protection and the smart substation technology advantages. This paper expounds the definition and features of the Agent, the characteristics of multi-Agent technology in relay protection and its substation-area protection. The substation-area protection can accelerate protect action time and realize the adaptive, which effectively solves the problems existing in the traditional relay protection. In the end, the article introduces the application prospect of the substation-area protection based on multi-Agent technology .
\end{abstract}

\section{Introduction}

Under the impetus of the strong smart grid construction, the application of intelligent substation has been rapid development, but its protection configuration still inherit the traditional characteristics of relay protection. Protection device is still configured based upon the object of protection, such as the main-transformer protection, bus protection, line protection[1]. Substation-area protection action at present is based on local measurement information. Due to measurement information is not real-time synchronization and sharing, protection devices in case of system failure and the operation mode change can not be effective coordination and it may malfunction or refuse to move, which result in the loss of protection selectivity and reliability. With the continuous development of distributed artificial intelligence and Internet communications technology, intelligent technology is becoming more and more attention in the application of relay protection. Agent is an intelligent unit, which is derived from distributed artificial intelligence. It can actively sense changes in ambient conditions and respond independently. Furthermore, multi-Agent technology has been the rapid development in recent years.

Aiming at the shortcomings of the current substation-area protection configuration, the paper researches on the characteristics and structures of relay protection and presents the smart substation-area protection based on multi-Agent technology. This protection can use the excellent protection features of Agent and intelligent substation technology, achieving real-time synchronization and sharing station panorama information, and optimizing the protection configuration of smart substation. This paper expounds its application prospect based on the research of the smart substation-area protection. 


\section{Multi-Agent System(MAS)}

Although having decades of development, Agent does not have a generally accepted concept. So far, one more authoritative definition is the concepts of "strong definition" and "weak definition", which was put forward by M.J. Wooldridge and N.R. Jennings in 1995 [3][4].As an intelligent unit, Agent can be used as an information detection unit and protective operation unit. We generally use multiple Agent be coordinated through a communication network to form a Multi-Agent System. Multi-Agent system can enhance the ability of the whole system to solve the problem by assigning tasks and coordinate cooperation. It solves these problems of the single Agent function incomplete and its information inaccurate[5][6].

MAS is a hierarchical distributed architecture, as shown in Figure 1, is divided into execution layer, coordination and decision-making layer. Multiple Agent according to its corresponding function distribution at the corresponding layers, such as collection Agent and tripping Agent in the execution layer, detection Agent and fault Agent in coordination layer, network reorganization Agent in decision-making layer.

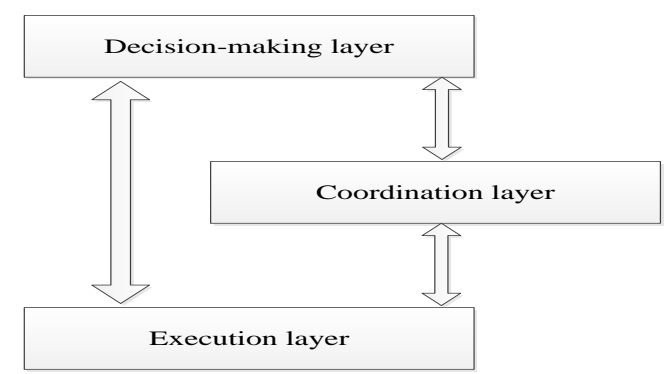

Figure 1 The basic structure of MAS

\section{Substation-area Protection Based On MAS}

Substation-area protection can provide protection functions to each of the station's equipment with the features of real-time synchronization and sharing of local information, which can be called smart substation domain protection[7].

The substation-area domain protective structure based on MAS is shown in figure 2. Center concentrative system in the figure adopted PCI-E bus technology, enabling real-time synchronization and gathering information, which can achieve the information real-time connectivity and synchronization between each Agent. MAS has the execution layer, coordination layer and decision-making layer. Each layer are located a number of different Agent, and its features are different.

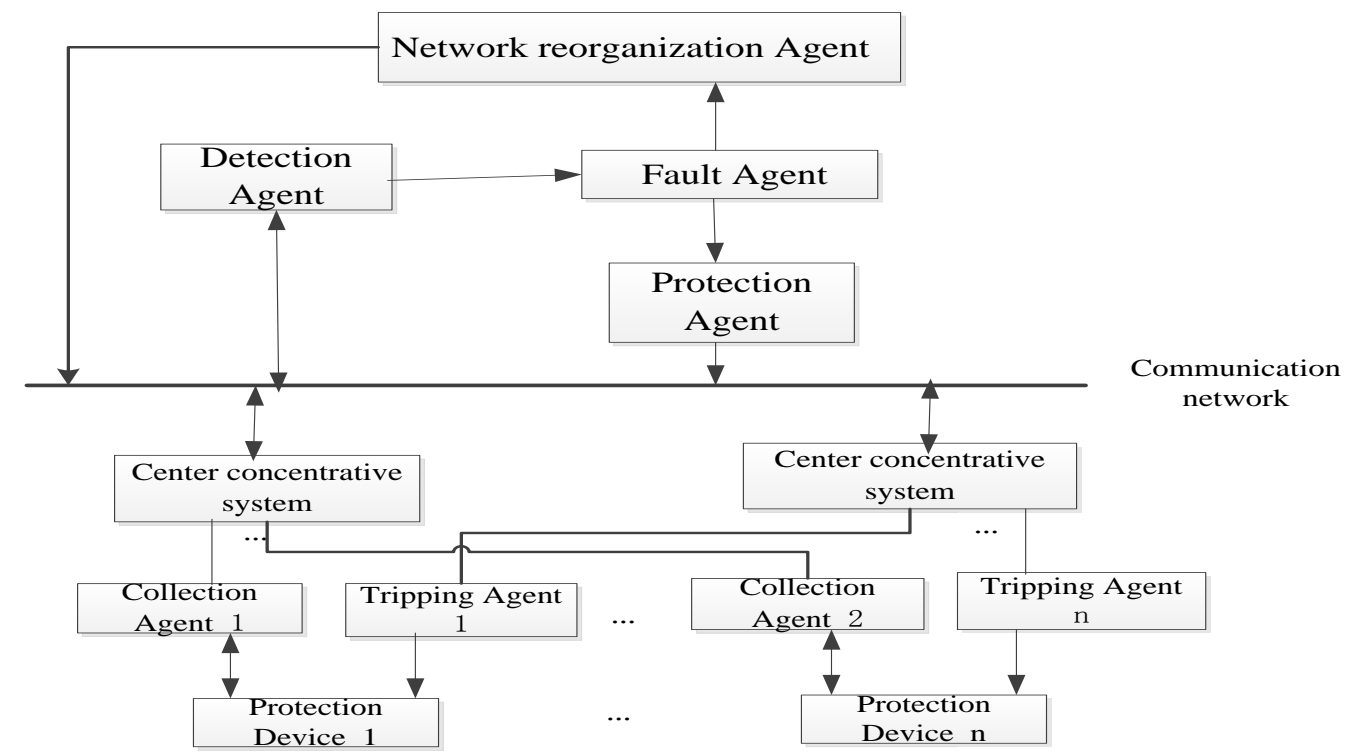

Figure 2 the substation-area protection structure based on MAS 
1) There are collection Agent and tripping Agent in the execution layer. The collection Agent can gather real-time information of conservation devices, such as the information of the Current Transformer(CT),Potential transformer(PT), overcurrent elements and circuit breaker operation. Tripping Agent can issue tripping command to the circuit breaker, and feed back this information to the detection Agent.

2) There are detection Agent, fault Agent and protection Agent in the coordination layer. The detection Agent monitor the real-time input information from the collection Agent, and feeds back this information to the fault Agent. Fault Agent applies the fault diagnosis algorithm based on the input information to locate the fault location. After fault cleared, the fault Agent feeds back this information to the network reorganization Agent. The protection Agent issues action command to the appropriate tripping Agent according to the fault location information.

3) There is a network reorganization Agent in decision-making layer. After receiving the fault cleared message, the network reorganization Agent network will re-draw the new substation-area network node graph. According to the new operation mode, the network reorganization Agent resets substation-area protection settings, and will send protection settings to the corresponding collection agents.

The system structure is shown in Figure 3,which is used to analyze the substation-area protection working principle simply.

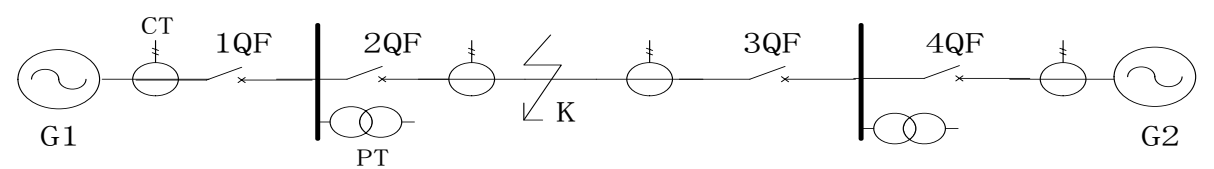

Figure 3 Dual power network simple schematic diagram

1) The substation-area operation is normal

The collection Agent sends real-time information of protection equipment to detection Agent, including overcurrent elements operation information and circuit breaker status information. The detection Agent monitor the real-time operation information, and judges according to this information.

2) There is a failure at $\mathrm{K}$ point

when a failure in $\mathrm{K}$ point is happened, the detection Agent detects changes in operating information, and sends a signal to the fault Agent. The fault Agent determines substation-area failure, and uses the fault location algorithms to judge fault location, which determines a failure occurs in the $\mathrm{K}$ point and immediately sends the fault location information to the protection Agent. After getting the fault information, the protection Agent issues immediately action commands to the tripping Agent to trip off the circuit breakers of 2QF and 3QF. After getting the command, the tripping Agent immediately act trip. After the fault is cleared, the detection Agent feed back new operational information to the fault Agent, and activate the network reorganization Agent to re-draw a new substation-area network node graph. According to the new operation mode, the network reorganization Agent will reset substation-area protection settings, and will send protection settings to the corresponding collection agents.

3) When a failure of $\mathrm{K}$ point is happening, the circuit breaker refuse to act.

After the tripping Agent issues immediately action commands to the circuit breakers of 2QF and 3QF,these breakers refuse to act. At the same time, the detection Agent immediately detects the circuit breakers of $2 \mathrm{QF}$ and $3 \mathrm{QF}$ have been in a closed state. After getting these information, the protection Agent based on selectivity of protection to derive the nearest circuit breaker are the circuit breakers of $1 \mathrm{QF}$ and $4 \mathrm{QF}$, and immediately issues a trip command to the tripping Agent. After receiving the command, the tripping Agent immediately issues tripping command to the circuit breakers, which can achieve acceleration protection action.

4) The fault is cleared by the actions of the circuit breakers

After detecting the changes of circuit breakers information, the detection Agent starts with the other Agent for information exchange. At the same time, fault Agent determines that the fault is cleared. After receiving the fault clearing messages, the network reorganization Agent resets 
substation-area protection settings, which can achieve the adaptation of protection.

\section{The application prospect of the substation-area protection based on multi-Agent technology}

Using network communications platform, the smart substation-area protection can achieve panorama information sharing and data synchronization. The protection based on multi-Agent technology, can achieve optimal protection principle and improve the accuracy of fault position location.

1) The protection can achieve optimal protection principle.

Through mutual coordination between the various Agent, it can accelerate protection action. In particular, it is better to improve the shortcomings of traditional backup protection operation time is too long, so as to realize backup protection without time limit for action. Due to it can obtain real-time information, the protection can simplify protection algorithm and cooperate relationship, which can online set so as to realize protection adaptive protection setting. In the literature [8], the substation-area protection is composed of current components and voltage components, its protection principle is simple and reliable. Backup protection based on multi-Agent technology can rapid clear fault and reset substation-area protection settings to improve the adaptability of protection.

2) The protection can improve the accuracy of fault position location.

Each collection Agent gathers real-time information on-site. When a failure is happening, the fault Agent analyzes the change information about CT, PT, circuit breakers and switches and etc. The fault Agent uses various fault judging principle to obtain quickly and accurately fault information, including fault location, fault type, fault phase and so on, which can improve the internal reliability of fault diagnosis and fault location accuracy[9]. In the literature [10], the protection can be based on these information to determine the fault zone and use fault judgment matrix algorithm to determine accurately the fault location after obtaining the change information from impedance elements and power directional elements by the data collection Agent, which can improve the flexibility and reliability of protection by using the distributed processing and cooperation ability between the various Agent.

\section{Conclusion}

Multi-Agent technology not only has strong intelligence, but also is a new kind of thinking mode. In this paper, the smart substation-area protection based on multi-Agent technology has carried on the preliminary research. At last, the article expounds the application prospect of the substation-area protection based on multi-Agent technology. However, the smart substation-area protection algorithm needs to be optimized in current time. Multi-Agent technology is lack of unified intelligent software system development and the evaluation criteria, which requires the continuous improvement in the future.

\section{References}

[1] Gao Dongxue, ZHI Quanzhong,Zhu Lijun, etc.Configuration project of protect unit according to levels for smart substation,Power System Protection and Control,2012,40(1): 69-70.

[2] Wong S K, Kalam A. An Agent Approach to Designing Protection Systems. In: IEE Sixth International Conference on Developments in Power System Protection. Nottingham (UKD :1997. $373 \sim 376$.

[3] Wooldridge M J, Jennings $\mathrm{N}$ R. Intelligent Agents: Theory and Practice. Knowledge Engineering Review, 1995, 10(2D : 115 152.

[4] ZHAN Hong-xia,YAN Quan.Application of Multi-agent Technology in Power System,Journal of Chongqing University(Natural Science Edition),2006,29(11):53-54. 
[5]Chen Yanxia, Yin Xianggen, Zhang Zhe, etc.multi-Agent system based protective relaying system,Automation of Electric Power Systems ,2002,26 (12): 1-5.

[6] HAN Xue-jun,ZHAO Feng-ying,SHI Lei,etc.Study on relaying protection based on the multi-agent system,Relay,2008,36(7):1-3.

[7]LIU Yiqing, GAO Houlei, LI Naiyong,etc.A communication scheme between smart substations for substation-area backup protection,Power System Protection and Control,2015,43(2):96-101.

[8]Wang Yuan, Jiao Yanjun.Research on substation-area protection system based on multi-Agent system,Power System Protection and Control,2013,41(3):80-83.

[9]BIAN Li, BIAN Chen-yuan.Review on intelligence fault diagnosis in power networks,Power System Protection and Control,2013,41(3):80-83.

[10]ZHAO Feng-xian;SUN Li-ying.Study on Regional Protection System of Microgrid Based on Multi-Agent System,Electric Power,2014,47(5):60-62. 